\title{
$\mathbb{A}$ Economics Bulletin
}

Volume 37, Issue 3

\section{Rationalizability of Choice Functions: Domain Conditions}

\author{
Debabrata Pal \\ Assistant Professor, Jawaharlal Nehru University, New Delhi, India
}

\begin{abstract}
In the literature related to choice theory an important problem which has been dealt with at length is the rationalizability of the choice function of an individual. In the literature a number of choice consistency conditions have been postulated which are proven to be necessary and sufficient for a choice function to have an ordering rationalization. In this paper a necessary and sufficient condition has been derived for the domain to be such that every possible choice function defined over the domain has an ordering rationalization.
\end{abstract}

Author wants to thank Prof. Satish K. Jain and Prof. Vicki Knoblauch for their comments and suggestions.

Citation: Debabrata Pal, (2017) "Rationalizability of Choice Functions: Domain Conditions", Economics Bulletin, Volume 37, Issue 3, pages 1911-1917

Contact: Debabrata Pal - debabrata1234@gmail.com.

Submitted: May 28, 2017. Published: August 31, 2017. 


\section{Introduction}

Domain conditions have played an important role in social choice theory ${ }^{1}$. Domain conditions were formulated under which the paradox of voting does not take place. These conditions restricted the set of admissible profiles of individual orderings. Black's (1948) single-peakedness condition provides an example.

Domain condition of a nature, as would be shown in this paper, is also relevant in the theory of rational choice ${ }^{2}$. The analysis of rational choice behaviour in the context of set valued choice functions gives rise to an interesting problem namely, the rationalizability of choice functions. The problem investigated is whether it is possible to find a preference relation which would generate the given choice pattern of an individual in different environments.

If it is observed that an individual chooses $x$ from $\{x, y\},\{x, z\},\{x, y, z\}$ and $y$ from $\{z, y\}$, it is immediate that the preference relation $x R x, y R y, x P y, x P z, y P z^{3}$ can generate such choice behaviour. The best element $(x)^{4}$ in the sets $\{x, y\},\{x, z\},\{x, y, z\}$ according to the aforesaid preference relation is the same as the chosen element in the sets. Similarly, $y$ is the best as well as the chosen element in the set $\{z, y\}$. We, therefore, say that such a choice function ${ }^{5}$ is rationalizable. In other words, a choice function is rationalizable if and only if it is possible to find a preference relation such that only the most preferred elements of a set according to that preference relation are chosen from that set.

The notion of rational choice, however, has been improvised further in the literature to capture different aspect of choice. Gaertner and Xu (2004) tries to incorporate the procedural aspect of choice where the available alternatives are linked to a procedure by which they came into existence. Bossert et al. (2005) invokes the notion of maximal-element ${ }^{6}$ rationalizability that requires an existence of a preference relation such that chosen elements are same as the maximal elements for every set in the domain according to that preference relation. Manzini and Mariotti (2007), Hung Au and Kawai (2011) consider an environment where choices are made sequentially. Under such consideration a decision maker uses more than one preference relations in a fixed order to remove non-preferred alternatives. This procedure sequentially rationalizes the choice function of the decision maker if a unique choice is made for every set belonging to the domain. Apesteguia and Ballester (2013) also considers choices by sequential procedure wherein a decision maker makes a choice by ruling out inferior alternatives through binary comparisons in a particular order.

Going by the definition of rationalizability as discussed above It may be noticed that not all choice functions are rationalizable ${ }^{7}$. In the literature a number of choice consistency conditions thus have been introduced, which ensure the rationalizability of

\footnotetext{
${ }^{1}$ For an illuminating discussion on domain conditions in social choice theory, see Gaertner (2001).

${ }^{2}$ Rational choice requires that choice behaviour is purposive and consistent.

${ }^{3}$ Read $x R x$ as ' $x$ is at least as good as $x$ ' and $x P y$ as ' $x$ is preferred to $y$ '.

${ }^{4}$ The definition of best element has been given in the next section.

${ }^{5}$ Here we use the phrases 'choice function' and 'choice pattern' interchangeably and with the same interpretation. The formal definition of choice function is given in the next section.

${ }^{6} x$ is said to be a maximal element of a set $S$ with respect to a preference relation $R$ iff no element in $S$ is preferred to $x$.

${ }^{7}$ Consider following choice function: $X=\{x, y, z\}, C(\{x, y\})=\{x\}, C(\{x, z\})=\{z\}, C(\{z, y\})=\{y\}, C(\{x, y, z\})=\{x\}$, where $X$ is the set of alternatives. This choice function is not rationalizable.
} 
choice functions. It has been established that choice functions defined over the general domain ${ }^{8}$ have ordering rationalization if and only if they satisfy the Houthakker axiom of revealed preference (HOA). Choice functions defined over the full domain have ordering rationalization if and only if they satisfy Arrow's axiom (AA) ${ }^{9}$. Bossert et al. (2005) has introduced conditions- Direct Exclusion and Direct Irreversibility which have been proven to be necessary and suffient for Maximal element rationalizability of a choice function. Likewise, Manzini and Mariotti (2007) and Hung Au and Kawai (2011) have introduced Weak WARP and No Binary Chain Cycles Axiom (NBCC) in the context of sequential rationalizable choice respectively.

The nature of these choice consistency conditions is such that they impose restrictions on the choice behaviour of an individual and implications of choice consistency conditions also change as the domain of the choice function changes ${ }^{10}$.

Furthermore, if there exists a domain over which all choice functions are rationalizable i.e., in whatever way an individual makes her choice it always becomes rational then it seems difficult to find any meaningful interpretation of 'purposive behaviour' of an individual in that particular domain, which, as discussed before, is at the core of the notion of rational choice. It is, therefore, worth investigating the nature of domains in relation to the rationalizability of the choice function.

In this paper we shall introduce a domain condition C.1 and show that this condition is necessary and sufficient for a domain over which all choice functions have ordering rationalization, which in turn provides complete characterization of domain for ordering rationalizability. This paper is divided into four sections. Second section contains basic notations and definitions which have been used in the succeeding sections. Section three provides the characterization result. Section four concludes the paper.

\section{Notations And Definitions}

Let $X$ be a non-empty finite set of alternatives and $2^{X}$ the power set of $X$. For a set $S$, \# $S$ denotes the cardinality of the set $S$. Let $D$ be a nonempty collection of nonempty subsets of $X, D \subseteq 2^{X}-\{\emptyset\}$. A choice function $C$ is a mapping from $D$ to $2^{X}-\{\emptyset\}$, $C: D \mapsto 2^{X}-\{\emptyset\}$ such that $C(S) \subseteq S$ for all $S \in D$.

Let $R$ be a binary relation defined over $X$. Let $I$ and $P$ denote symmetric and asymmetric parts of $R$ respectively. $R$ defined on $S$ is said to be reflexive iff $(\forall x \in S)(x R x)$

connected iff $(\forall x, y \in S)(x \neq y \rightarrow x R y \vee y R x)$

transitive iff $(\forall x, y, z \in S)(x R y \wedge y R z \rightarrow x R z)$

quasi-transitive iff $(\forall x, y, z \in S)(x P y \wedge y P z \rightarrow x P z)$.

We say that $R$ is an ordering iff it is reflexive, connected and transitive. It is a quasi-ordering iff it is reflexive and transitive. Let $R$ and $R^{\prime}$ be binary relations on a set S. $R^{\prime}$ is called an extension of $R$ iff $\left[R \subseteq R^{\prime} \wedge P(R) \subseteq P\left(R^{\prime}\right)\right]$.

Define binary relation $R_{c}$

$$
R_{c}=\{(x, y) \in X \times X \mid(\exists S \in D)(x \in C(S) \wedge y \in S)\} .
$$

\footnotetext{
${ }^{8}$ The general domain is a nonempty collection of nonempty subsets of the set of alternatives. The full domain is the collection of all nonempty finite subsets of the set of alternatives.

${ }^{9}$ See: $\operatorname{Arrow}(1959)$, Suzumura(1983).

${ }^{10}$ Take AA for instance, under full domain AA is necessary and sufficient for a choice function to have ordering rationalization. When the domain is not full AA fails to be sufficient for ordering rationalization.
} 
$x$ is said to be a greatest element (best) in a set $S$ with respect to a binary relation $R$ iff $(\forall y \in S)(x R y)$. Let $G(S, R)$ denote the set of greatest elements of a set $S$ with respect to $R$.

\section{Rationalizability and Domain Condition}

We introduce domain condition C.1. We first establish that this condition is necessary and sufficient for a domain over which every choice function has transitive rationalization and subsequently we show that the same condition is necessary and sufficient for domains over which every choice function has ordering rationalization.

C.1: $\forall n \in N-\{1\}, \forall$ distinct $x_{o}, x_{1}, x_{2}, \ldots, x_{n} \in X$, and $\forall S_{1}, S_{2}, \ldots, S_{n} \in D$, it should not be the case that $\left[S_{1} \neq S_{n} \wedge\left(\left\{x_{o}, x_{1}\right\} \subseteq S_{1} \wedge\left\{x_{1}, x_{2}\right\} \subseteq S_{2} \wedge \ldots . \wedge\left\{x_{n-1}, x_{n}\right\} \subseteq\right.\right.$ $\left.S_{n}\right)$ and $\left.\left(\exists S^{\prime} \in D\right)\left(\left\{x_{o}, x_{n}\right\} \subseteq S^{\prime}\right)\right]$

This condition requires that for distinct elements $x_{o}, x_{1}, x_{2}, \ldots, x_{n}$ and sets $S_{1}, S_{2}, \ldots, S_{n}$, where $S_{1} \neq S_{n}$, if it is the case that $x_{o}, x_{1}$ belong to $S_{1}, x_{1}, x_{2}$ belong to $S_{2}$, and so on and $x_{n-1}, x_{n}$ belong to $S_{n}$ then it would not be the case that there exists a set $S^{\prime}$ such that $x_{o}, x_{1}$ belong to that set. The underlying intuition of this condition is if we have a chain like $\left\{x_{o}, x_{1}\right\} \subseteq S_{1},\left\{x_{1}, x_{2}\right\} \subseteq S_{2}, \ldots \ldots,\left\{x_{n-1}, x_{n}\right\} \subseteq S_{n}$ and have a choice function such that following chain is obtained $x_{o} R_{c} x_{1}, x_{1} R_{c} x_{2}, x_{2} R_{c} x_{3}, \ldots, x_{n-2} R_{c} x_{n-1}, x_{n-1} R_{c} x_{n}$ then existence of a set $S^{\prime}$ containing element $x_{o}, x_{n}$ may give rise to the case $\left\{x_{n}\right\}=C\left(S^{\prime}\right)$ which would ensure, by the virtue of transitivity, that $x_{o}$ belongs to the set of best elements of $S^{\prime}$ with respect to $R_{c}$ but $x_{o} \notin C\left(S^{\prime}\right)$. The condition C.1 prevents such cases.

\subsection{Transitive Rationalizability and Domain Condition}

In the previous section we have defined binary relation $R_{c}$. We now define following binary relations:

$$
\begin{gathered}
R_{2}=\left\{(x, z) \in X \times X \mid\left(\exists y_{1}, y_{2}, . ., y_{n} \in X\right)\left(x R_{c} y_{1} \wedge y_{1} R_{c} y_{2} \wedge \ldots \wedge\right.\right. \\
\left.\left.y_{n} R_{c} z\right) \wedge(\forall T \in D)(\{x, z\} \nsubseteq T), \text { for some } n \in N\right\} \\
\bar{R}=R_{c} \cup R_{2}
\end{gathered}
$$

Lemma: Let choice function $C$ be defined over $D$. If $D$ satisfies condition $C .1$ then $\forall n \in N, \forall$ distinct $x_{o}, x_{1}, x_{2}, \ldots, x_{n} \in X:\left[x_{o} R_{c} x_{1} \wedge x_{1} R_{c} x_{2} \wedge x_{2} R_{c} x_{3} \wedge \ldots . \wedge x_{n-1} R_{c} x_{n} \rightarrow\right.$ $\left.x_{o} \bar{R} x_{n}\right]$.

Proof:

Let choice function $C$ be defined over $D$ and $D$ satisfy condition $C$.1. Let $x_{o}, x_{1}, x_{2}, \ldots, x_{n} \in$ $X$ be distinct, for some $n \in N$, and $x_{o} R_{c} x_{1} \wedge x_{1} R_{c} x_{2} \wedge x_{2} R_{c} x_{3} \wedge \ldots . \wedge x_{n-1} R_{c} x_{n}$.

If $n=1$ then $x_{o} R_{c} x_{n}$ is immediate and hence $x_{o} \bar{R} x_{n}$ by definition of $\bar{R}$.

Let $n \in N-\{1\}$.

$$
x_{i-1} R_{c} x_{i} \rightarrow\left(\exists S_{i} \in D\right)\left(x_{i-1} \in C\left(S_{i}\right) \wedge x_{i} \in S_{i}\right), \text { for } 1 \leq i \leq n .
$$

If $x_{n} \in S_{1}$ then we have $x_{o} R_{c} x_{n}$ and hence $x_{o} \bar{R} x_{n}$.

$x_{n} \notin S_{1} \rightarrow S_{1} \neq S_{n}$

$C .1 \rightarrow\left(\nexists S^{\prime \prime} \in D\right)\left(\left\{x_{o}, x_{n}\right\} \subseteq S^{\prime \prime}\right)$ 
$\rightarrow x_{o} R_{2} x_{n}$

$\rightarrow x_{o} \bar{R} x_{n}$

Theorem 1: Every choice function defined over $D$ has a transitive rationalization iff $D$ satisfies condition C.1.

Proof:

Suppose $D$ violates condition $C$.1, i.e.,

$\exists n \in N-\{1\}, \exists$ distinct $x_{o}, x_{1}, x_{2}, \ldots, x_{n} \in X$ and $\exists S_{1}, S_{2}, \ldots, S_{n} \in D$ such that $\left(S_{1} \neq\right.$ $\left.S_{n} \wedge\left(\left\{x_{o}, x_{1}\right\} \subseteq S_{1} \wedge\left\{x_{1}, x_{2}\right\} \subseteq S_{2} \wedge \ldots . \wedge\left\{x_{n-1}, x_{n}\right\} \subseteq S_{n}\right) \wedge\left(\exists S^{\prime} \in D\right)\left(\left\{x_{o}, x_{n}\right\} \subseteq S^{\prime}\right)\right)$.

Now we have four cases to consider:

(i) $S_{1}=S^{\prime}$; (ii) $S_{n}=S^{\prime}$; (iii) $S_{i}=S^{\prime}$, for some $i \in\{2,3,4, \ldots, n-1\}$; (iv) $S^{\prime} \neq S_{i}$, for $i \in\{1,2,3 \ldots, n\}$.

It is given that $\left\{x_{o}, x_{1}\right\} \subseteq S_{1} \wedge\left\{x_{1}, x_{2}\right\} \subseteq S_{2} \wedge \ldots \ldots \wedge\left\{x_{n-1}, x_{n}\right\} \subseteq S_{n}$.

Define the sets $P_{1}, P_{2}, \ldots, P_{n}$ in the following way:

$P_{1}=\left\{x_{o}, x_{1}\right\} \subseteq S_{1} \wedge P_{2}=\left\{x_{1}, x_{2}\right\} \subseteq S_{2} \wedge . . \wedge P_{i}=\left\{x_{i-1}, x_{i}\right\} \subseteq S_{i} \wedge \ldots \wedge P_{n}=\left\{x_{n-1}, x_{n}\right\} \subseteq$ $S_{n}$ Define, $S_{i}^{*}=\left\{P_{j} \mid S_{j}=S_{i}\right.$, for $\left.j \in\{1,2, \ldots, n\}\right\}$, for $i \in\{1,2, \ldots, n\}$.

Case (i): Let $S_{1}=S^{\prime}$.

Consider the following choice function: $\tilde{C}\left(S_{i}\right)=\bigcup_{P_{j} \in S_{i}^{*}} P_{j}$, for $i \in\{1,2, \ldots, n\}$

This choice function does not have any transitive rationalization.

Case (ii): Let $S_{n}=S^{\prime}$.

With the help of previous example we can show that there exists a choice function which does not have any transitive rationalization.

Case (iii): Let $S_{i}=S^{\prime}$ for some $i \in\{2,3,4, \ldots, n-1\}$.

If $\left\{x_{o}, x_{n}\right\} \subseteq S_{1} \vee\left\{x_{o}, x_{n}\right\} \subseteq S_{n}$ then previous cases hold again.

Let $\sim\left(\left\{x_{o}, x_{n}\right\} \subseteq S_{1} \vee\left\{x_{o}, x_{n}\right\} \subseteq S_{n}\right)$.

With the help of previous example we can show that there exists a choice function which does not have any transitive rationalization.

Case (iv): Let $S^{\prime} \neq S_{i}$ for $i \in\{1,2,3 \ldots, n\}$.

Consider the following choice function:

$\tilde{C}\left(S_{i}\right)=\bigcup_{P_{j} \in S_{i}^{*}} P_{j}$, for $i \in\{1,2, \ldots, n\}$; and $\tilde{C}\left(S^{\prime}\right)=\left\{x_{n}\right\}$

This choice function does not have any transitive rationalization.

Let $D$ satisfy condition $C$.1. Let $C$ be any choice function defined over $D$. We show that $\bar{R}$ rationalizes choice function $C$ i.e., we show that $C(S)=G(S, \bar{R})$.

Let $x \in C(S)$

$\rightarrow(\forall y \in S)\left(x R_{c} y\right)$

$\rightarrow x \in G(S, \bar{R})$ by definition of $\bar{R}$.

Let $x \in G(S, \bar{R})$.

Suppose $x \notin C(S)$

$\rightarrow(\exists y \in S)(y \in C(S))$

$\rightarrow\{x, y\} \subseteq S$.

$x \in G(S, \overline{\bar{R}}) \rightarrow(\forall z \in S)(x \bar{R} z)$.

Since $(\forall z \in S)(\{x, z\} \subseteq S)$

$\rightarrow(\forall z \in S)\left(\sim x R_{2} z\right)$

$\rightarrow(\forall z \in S)\left(x R_{c} z\right)$

$\rightarrow(\exists T \in D-\{S\})(\{x, y\} \subseteq T)$.

It is evident that $x, y$ are distinct elements and $S, T$ are distinct sets. 
$\rightarrow \# S \geq 3 \vee \# T \geq 3$

$\rightarrow(\exists x, y, z \in X)\left(\exists S_{1}, S_{2} \in D\right)\left(x, y, z\right.$ are distinct $\wedge S_{1} \neq S_{2} \wedge\left(\{x, y\} \subseteq S_{1} \wedge\{y, z\} \subseteq\right.$ $\left.\left.S_{2}\right) \wedge\left(\exists S^{\prime} \in D\right)\left(\{x, z\} \subseteq S^{\prime}\right)\right)$.

This implies violation of condition C.1.

Now, we show that $\bar{R}$ is transitive.

Let $x, y, z \in X \wedge(x \bar{R} y \wedge y \bar{R} z)$.

There are four cases to consider: (a) $x R_{c} y \wedge y R_{c} z,(b) x R_{c} y \wedge y R_{2} z,(c) x R_{2} y \wedge y R_{c} z$, (d) $x R_{2} y \wedge y R_{2} z$.

Case (a): Let $x, y, z \in X \wedge\left(x R_{c} y \wedge y R_{c} z\right)$.

If $x=y$ or $y=z$ then $x R_{c} z$ follows immediately. If $x=z$ then $x R_{c} z$ follows from the definition of $R_{c}$.

Let $x, y, z$ be distinct elements.

$x R_{c} y \rightarrow(\exists S \in D)(x \in C(S) \wedge y \in S)$.

$y R_{c} z \rightarrow(\exists T \in D)(y \in C(T) \wedge z \in T)$.

If $\{y, z\} \subseteq S$ then $x R_{c} z$ follows by definition of $R_{c}$.

Suppose $\{y, z\} \nsubseteq S$.

$\rightarrow S, T$ are distinct sets.

Suppose $\left(\exists S^{\prime \prime} \in D\right)\left(\{x, z\} \subseteq S^{\prime \prime}\right)$

$\rightarrow$ violation of condition $C .1$.

Let $\sim\left(\exists S^{\prime \prime} \in D\right)\left(\{x, z\} \subseteq S^{\prime \prime}\right)$.

$\rightarrow(x, z) \in R_{2}$

$\rightarrow x \bar{R} z$.

Case (b): Let $x, y, z \in X \wedge\left(x R_{c} y \wedge y R_{2} z\right)$.

$y R_{2} z \rightarrow\left(\exists w_{2}, w_{3}, \ldots, w_{n-1} \in X\right)\left(y R_{c} w_{2} \wedge w_{2} R_{c} w_{3} \wedge \ldots \wedge w_{n-2} R_{c} w_{n-1} \wedge w_{n-1} R_{c} z\right)$.

So we have: $x R_{c} y \wedge y R_{c} w_{2} \wedge w_{2} R_{c} w_{3} \wedge \ldots \wedge w_{n-2} R_{c} w_{n-1} \wedge w_{n-1} R_{c} z$.

Now if $z=y \vee z=x$ then $x R_{c} z$ is immediate and hence $x \bar{R} z$.

If $y=x$ then $x R_{2} z$ is immediate and hence $x \bar{R} z$.

Suppose $z, x, y$ are distinct.

$(1) \wedge(2)$ imply that there exists a following chain of distinct elements.

$x R_{c} v_{1} \wedge v_{1} R_{c} v_{2} \wedge v_{2} R_{c} v_{3} \wedge \ldots \wedge v_{m-2} R_{c} v_{m-1} \wedge v_{m-1} R_{c} z$, for $m \leq n \in N$.

Hence by lemma we have $x \bar{R} z$.

For Case (c) and Case (d), showing $x \bar{R} z$ is analogous to the Case (b).

Hence the theorem is established.

\subsection{Ordering Rationalizability and Domain Condition}

Before we state and prove the result we introduce following definitions:

Define $\triangle_{X}$ as follows: $\triangle_{X}=\{(x, x) \mid x \in X\}$.

We have defined $R_{2}$ in the previous theorem. Now define $R_{3}: R_{3}=\triangle_{X} \cup R_{c} \cup R_{2}$

Theorem 2: Every choice function defined over $D$ has an ordering rationalization iff $D$ satisfies condition C.1.

Proof:

In Theorem 1 we have shown if all choice functions defined over a domain have a transitive rationalization then the domain satisfies condition C.1. We show that if domain of choice functions satisfies condition $C .1$ then every choice function defined on that domain is rationalizable by a reflexive, connected and transitive preference relation.

In the previous theorem it has been established that $R_{c} \cup R_{2}$ is transitive. Hence $R_{3}$ 
is reflexive and transitive i.e., quasi-ordering. This implies that there exists an ordering extension of $R_{3}{ }^{11}$.

Let $\bar{R}$ be an ordering extension of $R_{3}$. We show that $\bar{R}$ rationalizes choice function. Let $C$ be any choice function defined over $D$ which satisfies condition $C .1$.

Let $S \in D$. We show: $C(S)=G(S, \bar{R})$.

Let $x \in C(S)$

$\rightarrow(\forall y \in S)\left(x R_{c} y\right)$

$\rightarrow(\forall y \in S)\left(x R_{3} y\right)$.

Since $\bar{R}$ is an extension of $R_{3}$

$\therefore(\forall y \in S)(x \bar{R} y)$

$\rightarrow x \in G(S, \bar{R})$.

Let $x \in G(S, \bar{R})$.

Suppose $x \notin C(S)$

$\rightarrow(\exists y \in S-\{x\})(y \in C(S))$.

$\rightarrow y R_{c} x$

$\rightarrow y R_{3} x$

$\rightarrow y I\left(R_{3}\right) x \vee y P\left(R_{3}\right) x$.

Since $\{x, y\} \subseteq S$, we have $\sim\left(x R_{2} y \vee y R_{2} x\right)$

$\rightarrow\left[\left(y I\left(R_{3}\right) x \rightarrow y I\left(R_{c}\right) x\right) \wedge\left(y P\left(R_{3}\right) x \rightarrow y P\left(R_{c}\right) x\right)\right]$.

Now there are two cases to consider: $(i)$ yI $\left(R_{3}\right) x(i i)$ yP $\left(R_{3}\right) x$.

Case (i): Suppose $y I\left(R_{3}\right) x$

$\rightarrow y I\left(R_{c}\right) x$

$\rightarrow y I(\bar{R}) x$

$(3) \wedge(4) \rightarrow(\exists T \in D-\{S\})(x \in C(T) \wedge y \in T)$.

It is evident that $x, y$ are distinct elements and $S, T$ are distinct sets.

$\rightarrow \# S \geq 3 \vee \# T \geq 3$

$\rightarrow(\exists x, y, z \in X)\left(\exists S_{1}, S_{2} \in D\right)\left(x, y, z\right.$ are distinct $\wedge S_{1} \neq S_{2} \wedge\left(\{x, y\} \subseteq S_{1} \wedge\{y, z\} \subseteq\right.$ $\left.\left.S_{2}\right) \wedge\left(\exists S^{\prime} \in D\right)\left(\{x, z\} \subseteq S^{\prime}\right)\right)$.

This implies violation of condition C.1.

Case (ii): Suppose $y P\left(R_{3}\right) x$

$\rightarrow y P(\bar{R}) x$, since $\bar{R}$ is an extension of $R_{3}$.

Again, $x \in G(S, \bar{R}) \rightarrow(\forall z \in S)(x \bar{R} z)$

$\rightarrow x \bar{R} y$

$(5) \wedge(6)$ lead to a contradiction.

Hence $x \in C(S)$.

\section{Conclusion}

Domain conditions in the context of rationalizability of choice function are important, not only because they provide a new set of conditions for rationalizability but also because they do not constrain the 'act of choice' or the choice behaviour of an individual. Unlike choice consistency conditions which characterize the partition between two classes of choice functions: rationalizale choice functions and nonrationalizable choice functions, domain conditions make a partition of domains. On one side there is a class of domains over which any choice function is rationalizable and on the other side there is a class of

\footnotetext{
${ }^{11}$ See: Szpilrajn, E (1930).
} 
domains over which not all choice functions are rationalizable. These domain conditions characterize the partitions of the domains. This paper provides complete characterization domains for ordering rationalizability. Full characterizations of quasitransitive and acyclic rationalizability are yet to be obtained.

\section{References}

Apesteguia, J., Ballester, M.A. (2013), Choice by Sequential Procedures. Games and Economic Behavior 77, 90-99.

Arrow, K. J. (1959), Rational Choice Functions and Orderings. Economica, New Series, 26, 102, 121-127.

Black, Duncan (1948), On the Rationale of Group Decision-making. Journal of Political Economy 56, 23-34.

Bossert,Walter, Sprumont, Yves and Suzumura, Kotaro (2005), Maximal-Element Rationalizability, Theory and Decision, 58, 325-350.

Gaertner, w., Domain Condition in Social Choice Theory, (2001). Cambridge University Press, New York.

Gaertner, w., Xu, Y. (2004), Procedural Choice. Economic Theory 24, 335-349.

Houthakker, H. S. (1950), Revealed Preference and the Utility Function. Economica, New Series, 17, 66, 159-174.

Hung, A.P., Kawai, K., J. (2011), Sequentially Rationalizable Choice with Transitive Rationales. Games and Economic Behavior 73, 608-614.

Manzini, P., Mariotti, M. (2007), Sequentially Rationalizable Choice. Amer. Econ. Rev. 97, 1824-1839.

Richter, M. K., Revealed Preference Theory. Econometrica (1966), 34, 3, 635-645.

Samuelson, P. A. (1938), A Note on the Pure Theory of Consumer's Be- haviour. Economica, New Series, 5, 17, 61-71.

Samuelson, P. A. (1950), The Problem of Integrability in Utility Theory. Economica, New Series, 17, 68, 355-385.

Suzumura, K., Rational Choice, Collective Decisions and Social Wel- fare (1983). Cambridge University Press, New York.

Szpilrajn, E. (1930), 'Sur l'Extension de l'Ordre Partiel', Fundamenta Mathematicae, 16:386-9. 